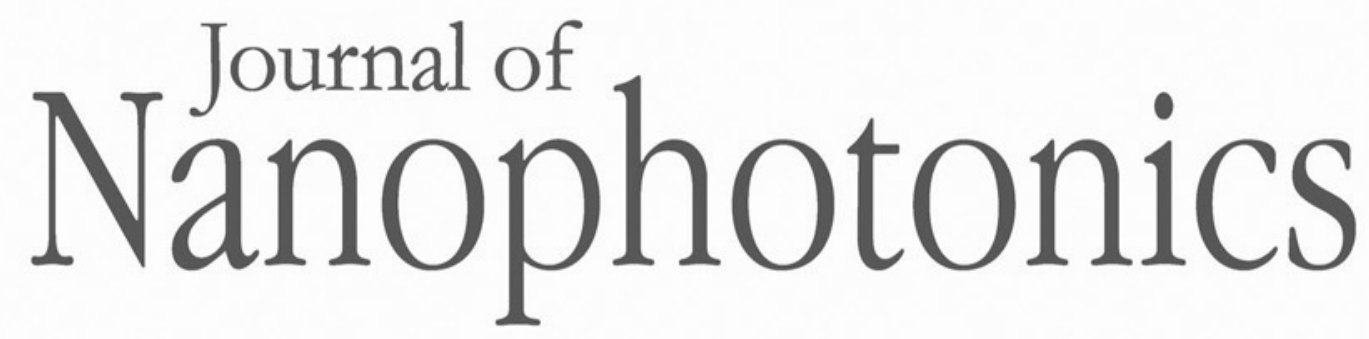

\title{
Special Section Guest Editorial: Nanoplasmonics for Biosensing and Enhanced Light-Matter Interaction
}

\author{
Junpeng Guo \\ Joshua R. Hendrickson \\ Ting S. Luk
}




\title{
Special Section Guest Editorial: Nanoplasmonics for Biosensing and Enhanced Light-Matter Interaction
}

\author{
Junpeng Guo, ${ }^{a}$ Joshua R. Hendrickson, ${ }^{\mathrm{b}}$ and Ting S. Luk ${ }^{\mathrm{c}}$ \\ ${ }^{a}$ University of Alabama in Huntsville, Nano and Micro Devices Center and Department of \\ Electrical and Computer Engineering, Huntsville, Alabama, United States \\ ${ }^{\mathrm{b}}$ Air Force Research Laboratory, Sensors Directorate, Wright-Patterson Air Force Base, Ohio, \\ United States \\ ${ }^{\mathrm{c} S a n d i a}$ National Laboratories, Center of Integrated Nanotechnologies, Albuquerque,
} New Mexico, United States

We are living in an era of nanotechnologies. Nanoscale behaviors are relevant to many science and technology applications. The ability to bring free-space electromagnetic optical radiations to the nanoscale through the use of plasmonic nanostructures is truly remarkable. Through optical field enhancement and geometrical structuring, enhanced light-matter interaction and biosensing are the most important applications. In this issue, Karim, Smith, and Zhao provide an in-depth and comprehensive review on single molecular detection using plasmonic nanostructures. In another paper, gold core-shell ultra-luminescent nanoparticles were shown to be useful as a new platform for biosensing and tracking applications. In this issue, three papers are on the topic of enhanced light absorption using plasmonic nanostructures. One of the papers discussed using controlled surface plasmon resonance enhanced light absorption in gold nanoparticles to generate heating-cooling cycles. The heating-cooling cycles in turn generate acoustic waves propagating in tissues for bio-imaging applications.

This special section of the Journal of Nanophotonics is dedicated to increasing the communication among scientists to share their thoughts, discoveries, and challenges in this emerging field.

\section{Acknowledgments}

The guest editors thank all authors for their contributions to this special section of the journal and also thank all reviewers and editorial staff members who helped put this work together.

Junpeng Guo is currently professor of electrical engineering and optics at the University of Alabama in Huntsville (UAH). Before his current position, he was a research scientist at the Rockwell International Science Center and a member of technical staff at Sandia National Laboratories. He received his $\mathrm{PhD}$ degree in electrical engineering from the University of Illinois at Urbana-Champaign. He was a recipient of the Alan Berman Research Publication Award from the Naval Research Laboratory (NRL) in 2013, and he was the only recipient of the University Distinguished Faculty Research Award at UAH in 2016. Currently, he serves as an associate editor of Journal of Nanophotonics and also serves as an associate editor of Photonics Research.

Joshua R. Hendrickson is a senior research physicist at the Air Force Research Laboratory, Sensors Directorate at Wright-Patterson Air Force Base, Ohio. He received his PhD degree in optical sciences from the University of Arizona. He was a recipient of the AFRL Early Career Award and the AFRL S\&T Award in 2015, and he also received the AFOSR STAR Team Award in 2017.

Ting S. Luk is a scientist at the Center of Integrated Nanotechnologies (CINT) and a principal member of technical staff at Sandia National Laboratories. He received his PhD degree in physics from the State University of New York at Stony Brook. He was a research professor at the

(C) 2017 Society of Photo-Optical Instrumentation Engineers (SPIE) 
University of Illinois at Chicago, where he was elected as a Fellow of the Optical Society of America in 1993. After a stint at the University of Wyoming as an assistant professor, he joined Sandia National Laboratories in 1999 and also became a CINT scientist in 2007. 